\title{
Novos sedativos hipnóticos
}

\section{The newer sedative-hypnotics}

\author{
Lucia Sukys-Claudino1, Walter André dos Santos Moraes ${ }^{1}$, Sergio Tufik ${ }^{1}$, Dalva Poyares ${ }^{1}$ \\ ${ }^{1}$ Departamento de Psicobiologia, Universidade Federal de São Paulo (UNIFESP), São Paulo, SP, Brasil
}

\begin{abstract}
Resumo
Nas últimas décadas houve um esforço para o desenvolvimento de hipnóticos mais seguros e eficazes. Zolpidem, zaleplona, zopiclona, eszopiclona (drogas-z) e indiplona são moduladores do receptor GABA-A, os quais agem de forma seletiva na subunidade $\alpha 1$, exibindo, desta forma, mecanismos similares de ação, embora evidências recentes sugiram que a eszopiclona não seja tão seletiva para a subunidade $\alpha 1$ quanto o zolpidem. Ramelteon e tasimelteon são novos agentes crono-hipnóticos seletivos para os receptores de melatonina MT1 e MT2. Por outro lado, nos últimos anos, o consumo de drogas antidepressivas sedativas tem aumentado significativamente no tratamento da insônia. Como droga experimental, a eplivanserina tem sido testada como um potente agonista inverso do subtipo $5-\mathrm{HT}_{2 \mathrm{~A}}$ da serotonina, com um uso potencial na dificuldade da manutenção do sono. Outro agente farmacológico para o tratamento da insônia é o almorexant, o qual apresenta um novo mecanismo de ação envolvendo antagonismo do sistema hipocretinérgico, desta forma levando à indução do sono. Finalmente, também discutiremos o potencial papel de outras drogas gabaérgicas no tratamento da insônia.
\end{abstract}

Descritores: Hipnóticos e sedativos; Efeitos fisiológicos de drogas; Receptores de melatonina; Antidepressivos; Distúrbios do início e da manutenção do sono

\begin{abstract}
There has been a search for more effective and safe hypnotic drugs in the last decades. Zolpidem, zaleplon, zopiclone, eszopiclone (the z-drugs) and indiplon are GABA-A modulators which bind selectively $\alpha 1$ subunits, thus, exhibiting similar mechanisms of action, although recent evidence suggests that eszopiclone is not as selective for $\alpha 1$ subunit as zolpidem is. Ramelteon and tasimelteon are new chrono-hypnotic agents, selective for melatonin MT1 and MT2 receptors. On the other hand, the consumption of sedative antidepressant drugs is significantly increasing for the treatment of insomnia, in the last years. As an experimental drug, eplivanserin is being tested as a potent antagonist of serotonin 2-A receptors (ASTAR) with a potential use in sleep maintenance difficulty. Another recent pharmacological agent for insomnia is almorexant, which new mechanism of action involves antagonism of hypocretinergic system, thus inducing sleep. Finally we also discuss the potential role of other gabaergic drugs for insomnia.
\end{abstract}

Descriptors: Hypnotics and sedatives; Physiological effects of drugs; Receptors, melatonin; Antidepressive agents; Sleep initiation and maintenance disorders

hipnótico-sedativas, desde o marco da síntese do zolpidem, incluindo os agonistas do receptor GABA-A Omega 1, agonistas do receptor da melatonina, agonistas gabaérgicos e outras drogas em desenvolvimento (Tabela 1).

Apesar de várias dentre elas terem sido aprovadas para uso, muitas das drogas apresentadas ainda não estão disponíveis para comercialização no Brasil.

\section{Hipnóticos de $2^{a}$ geração}

O zolpidem é uma imidazopiridina. Possivelmente, por ser o primeiro agonista seletivo do receptor GABA-A para a subunidade $\alpha 1$, foi apontado como o hipnótico mais prescrito no mundo ${ }^{6}$. Apresenta uma meia vida de 2,4 horas e não tem metabólitos ativos. Sua principal indicação é para rápida indução, com algum 
Tabela 1 - Mecanismo de ação, meia vida, metabolização e efeitos colaterais dos principais novos sedativos-hipnóticos

\begin{tabular}{|c|c|c|c|c|c|}
\hline & & Mecanismo de ação & $\begin{array}{c}1 / 2 \text { vida } \\
\text { aproximada }(h)\end{array}$ & Metabolização & $\begin{array}{l}\text { Principais efeitos } \\
\text { colaterais }\end{array}$ \\
\hline $\begin{array}{l}\text { Drogas-z } \\
\text { Hipnóticos de }\end{array}$ & Zolpidem* & $\begin{array}{l}\text { Receptor GABA-A } \\
\text { agonista } \alpha 1 \text { seletivo }\end{array}$ & 2,4 & $\begin{array}{l}\text { Hepática, sem metabólitos } \\
\text { ativos, mais lenta em idosos }\end{array}$ & $\begin{array}{l}\text { Sonolência, } \\
\text { cefaleia, cansaço }\end{array}$ \\
\hline \multirow[t]{2}{*}{$2^{a}$ geração } & Zaleplona & $\begin{array}{l}\text { Receptor GABA-A } \\
\text { agonista } \alpha 1 \text { seletivo }\end{array}$ & 0,9 & $\begin{array}{l}\text { Hepática, sem metabólitos } \\
\text { ativos }\end{array}$ & Cefaleia, tontura \\
\hline & Zopiclona & $\begin{array}{l}\text { Receptor GABA-A } \\
\text { agonista } \alpha 1 \text { e } a 2\end{array}$ & 5,3 & $\begin{array}{l}\text { Hepática, recomenda-se } \\
\text { redução da dose em } \\
\text { pacientes acima de } 70 \text { anos e } \\
\text { com insuficiência hepática }\end{array}$ & $\begin{array}{c}\text { Sabor metálico, } \\
\text { boca seca, tontura, } \\
\text { cefaleia, sonolência }\end{array}$ \\
\hline \multirow[t]{2}{*}{$\begin{array}{l}\text { Hipnóticos de } \\
3^{\text {a }} \text { geração }\end{array}$} & Eszopiclona & $\begin{array}{l}\text { Receptor GABA-A } \\
\text { agonista em sítios não } \\
\text { totalmente conhecidos }\end{array}$ & 5,8 & $\begin{array}{l}\text { Hepática, recomenda-se } \\
\text { ajuste de dose em pacientes } \\
\text { idosos e com insuficiência } \\
\text { hepática }\end{array}$ & $\begin{array}{c}\text { Sabor } \\
\text { desagradável, } \\
\text { cefaleia, náusea, } \\
\text { tontura, sonolência }\end{array}$ \\
\hline & Indiplona* & $\begin{array}{c}\text { Receptor GABA-A } \\
\text { agonista } \alpha 1 . \\
\text { Afinidade subunidade } \\
\alpha 6\end{array}$ & 1,5 & $\begin{array}{c}\text { Hepática, sem metabólitos } \\
\text { ativos }\end{array}$ & $\begin{array}{l}\text { Cefaleia, tontura, } \\
\text { sonolência, náusea }\end{array}$ \\
\hline \multirow[t]{2}{*}{ Melatoninérgicos } & Ramelteon & $\begin{array}{l}\text { Agonista receptores } \\
\text { MT1 e MT2 }\end{array}$ & $1-2$ & $\begin{array}{l}\text { Hepática (CYP1A2), com } \\
\text { metabólitos ativos até 2-5h. } \\
\text { Não deve ser usado com } \\
\text { inibidores da CYP1A2 } \\
\text { e insuficiência hepática }\end{array}$ & $\begin{array}{c}\text { Cefaleia, } \\
\text { sonolência, tontura }\end{array}$ \\
\hline & Melatonina* & $\begin{array}{l}\text { Receptores MT1 e } \\
\text { MT2 }\end{array}$ & $0,5-0,8$ & Hepática & Sonolência \\
\hline $\begin{array}{l}\text { Agonistas } \\
\text { gabaérgicos }\end{array}$ & Tiagabina & $\begin{array}{l}\text { Bloqueador do GAT-1, } \\
\text { transportador } \\
\text { específico do GABA }\end{array}$ & $7-9$ & Hepática, via citocromo $P_{450}$ & Tontura, náusea \\
\hline \multirow[t]{3}{*}{$\begin{array}{l}\text { Antidepressivos } \\
\text { sedativos }\end{array}$} & Doxepina & $\begin{array}{c}\text { Antidepressivo } \\
\text { tricíclico. } \\
\text { Antagonista } \mathrm{H1} \\
\text { específico quando em } \\
\text { baixas doses }\end{array}$ & 7,8 & Hepática & $\begin{array}{c}\text { Cefaleia, sonolência } \\
\text { (em baixas doses) }\end{array}$ \\
\hline & Agomelatina & $\begin{array}{l}\text { Agonista receptores } \\
\text { MT1 e MT2. }\end{array}$ & $2-3$ & Hepática (CYP1A2) & $\begin{array}{l}\text { Cefaleia, tontura, } \\
\text { náusea e fadiga }\end{array}$ \\
\hline & & $\begin{array}{c}\text { Antagonista } \\
\text { serotonina } 5 \mathrm{HT}_{2 \mathrm{C}}\end{array}$ & & & \\
\hline
\end{tabular}

* Existem duas formulações propostas: liberação imediata e liberação modificada.

efeito na consolidação sono. Sua eliminação é renal e está reduzida em pacientes com insuficiência renal crônica. A dose terapêutica média para insônia em adultos é de $10 \mathrm{mg}$ e de $5 \mathrm{mg}$ para idosos ${ }^{7,8}$.

A zopiclona é uma ciclopirrolona e difere do zolpidem por ter uma meia vida maior (5,3 horas) e por ser menos seletiva, atuando em receptores que contêm subunidades tanto $\alpha 1$ quanto $\alpha 2$. Apresenta maior potencial de sonolência residual pela manhã, e em estudos de eletroencefalografia induziu alteraçôes similares aos benzodiazepínicos ${ }^{8,9}$.

A zaleplona é uma pirazolopirimidina. Apresenta meia vida ultracurta (0,9 horas) e perfil de ligação ao receptor GABA-A similar ao zolpidem, sendo que sua principal indicação é para rápida indução de sono, com pouco efeito em sua manutenção, podendo ser utilizada no meio da noite, em casos de despertar precoce. A dose terapêutica também é de $10 \mathrm{mg}$ para adultos ${ }^{8,10}$.

$\mathrm{O}$ zolpidem e a zaleplona alteram pouco a estrutura do sono, são bem tolerados e estão pouco associados à ocorrência de tolerância e dependência ao uso prolongado. Ambos reduzem a latência para o início do sono e o zolpidem pode causar aumento adicional do tempo total de sono. Uma versão de liberação modificada do zolpidem foi sintetizada (MR), mantendo concentraçôes plasmáticas sustentadas no meio da noite, melhorando a manutenção do sono ${ }^{11}$. Um estudo multicêntrico publicado recentemente demonstrou segurança e eficácia no uso do zolpidem de liberação modificada três a sete vezes por semana por seis meses no tratamento da insônia crônica ${ }^{11}$.

\section{Hipnóticos de $3^{\mathrm{a}}$ geração}

1) Eszopiclona

Vários estudos têm sido publicados com eszopiclona, um isômero da zopiclona, aprovado pelo Food and Drug Administration (FDA) para tratamento da insônia. O Lunesta ${ }^{\circledR}$ foi o primeiro hipnótico liberado para uso em insônia crônica (tempo superior a um mês de duração) e está indicado tanto para a dificuldade em iniciar como para manter o sono. A dose recomendada é de $3 \mathrm{mg}$ para adultos e $2 \mathrm{mg}$ para idosos ${ }^{12}$.

Hanson et al. observaram que a eszopiclona pode exibir diferentes propriedades de ligação no receptor GABA-A, 
comparada com o zolpidem, além de ser menos seletiva para o receptor GABA-A que contém a subunidade $\alpha 1^{13}$.

A eszopiclona mostrou-se efetiva no tratamento da insônia crônica primária de acordo com os critérios do Manual Diagnóstico e Estatístico de Transtornos Mentais - 4a Edição - Texto Revisado (DSM-IV-R). Além disso, em um estudo duplo-cego, randomizado, placebo-controlado, melhorou o funcionamento durante o dia, por pelo menos seis meses, na dose de $3 \mathrm{mg}$, sem evidências de tolerância, dependência ou abuso. Entretanto, causou leves e transitórios problemas de memória em alguns pacientes ${ }^{14}$.

2) Indiplona

A indiplona é uma nova pirazolopirimidina com seletividade para receptores contendo a subunidade $\alpha 1$, e alguma seletividade para a subunidade $\alpha 6^{15}$. Um estudo com polissonografia mostrou que indiplona promove redução da latência do sono, do número de despertares após o início do sono, além de não apresentar efeitos residuais diurnos devido a sua meia-vida curta ${ }^{16}$.

A indiplona é rapidamente absorvida, atingindo níveis terapêuticos em torno de 30 minutos. É apresentada sob as formulações de liberação rápida (Indiplon IR) e controlada (Indiplon MR). A primeira possui efeito com duração média de quatro horas e está indicada para insônia com dificuldade para iniciar o sono, ou nos casos de despertares noturnos. A segunda, devido à duração média de efeito de sete horas, tem principal indicação nos casos de dificuldade de manutenção do sono ${ }^{17}$. Não foram observadas diferenças na farmacocinética da droga entre indivíduos jovens e idosos, tornando-se uma opção terapêutica para idosos com insônia crônica. As doses terapêuticas variam entre 15 e 30mg, nas formulações de liberação imediata ou controlada ${ }^{18}$.

\section{Melatoninérgicos}

1) Ramelteon

Trata-se de um agente agonista com alta seletividade para receptores de melatonina MT1 e MT2 localizados no núcleo supraquiasmático e que estão envolvidos na indução do sono e regulação do ciclo vigíliasono. Quase não possui afinidade por outros receptores tais como dopamina, serotonina, benzodiazepínicos, opioides e histamina. É rapidamente absorvido, com pico de ação de cerca de 30 minutos e meia-vida em torno de 1,2 horas. Existe relativo aumento da meiavida plasmática em indivíduos idosos, porém sem potencialização dos efeitos adversos ${ }^{19}$.

O Ramelteon não deve ser usado conjuntamente com fluvoxamina, assim como também em combinação com outros inibidores da CYP1A2, tais como ciprofloxacino e norfloxacino. A coadministração de ramelteon com cetoconazol e fluconazol ou indutores tais como rifampicina, carbamazepina ou barbitúricos deve ser evitada ${ }^{20}$.

Doses entre $8 \mathrm{mg}$ e $64 \mathrm{mg}$ promovem efeitos similares e significativa redução na latência e aumento no tempo total de sono sem, no entanto, alterar sua estrutura ${ }^{21}$.

Mayer et al. observaram segurança e boa tolerabilidade com baixa incidência de efeitos adversos durante o uso de ramelteon por um período de seis meses a um ano. O mesmo estudo não identificou potencial para indução de insônia de rebote, sintomas de abstinência, potencial para abuso e/ou dependência, comprometimento cognitivo ou motor, sugerindo ser uma opção terapêutica em pacientes com história prévia de abuso de substâncias e em pacientes idosos ${ }^{22}$.

A apresentação disponível é de comprimidos de $8 \mathrm{mg}$, os quais devem ser administrados 30 minutos antes de deitar.

2) Melatonina de liberação prolongada

A melatonina é um hormônio produzido pela glândula pineal, secretado no período noturno, e funciona como um regulador endógeno do ciclo sono-vigília.

Os efeitos crono-hipnóticos da melatonina exógena e de drogas melatoninérgicas são mediados via receptores MT1 e MT2, especialmente no núcleo supraquiasmático. A meia-vida de eliminação ultracurta $(0,5-0,8$ horas $)$ da melatonina é o maior obstáculo para seu uso no tratamento da insônia crônica primária, o que favoreceu o surgimento da formulação de liberação prolongada, Circadin ${ }^{23}$.

$\mathrm{O}$ uso da melatonina no tratamento dos distúrbios do ritmo circadiano já tem seu papel estabelecido conforme dados da American Academy of Sleep Medicine ${ }^{24}$. No entanto, no caso da insônia crônica primária, os resultados ainda não são tão consistentes na questão manutenção do sono, exceto em populações específicas, como, por exemplo, em pacientes acima de $55 \operatorname{anos}^{25}$. Segundo dados da literatura, a melatonina apresenta bom perfil de tolerabilidade e segurança, com poucos efeitos colaterais ${ }^{23,25}$.

A melatonina de liberação prolongada está disponível na apresentação de $2 \mathrm{mg}$, a qual pode ser administrada uma hora antes do horário de deitar.

\section{Agonistas GABA-A}

1) Tiagabina

A tiagabina é um bloqueador específico do GAT-1 transportador do GABA. É rapidamente absorvida após administração oral, com pico de ação em torno de 90 minutos. Apresenta ação anticonvulsivante, sendo seu uso aprovado para este fim desde $1997^{26}$.

Walsh et al. demonstraram que a tiagabina na dose de $4 \mathrm{mg}$ promove alteraçôes benéficas na arquitetura do sono. No entanto, doses acima de $8 \mathrm{mg}$ estão associadas à elevada incidência de efeitos adversos, efeitos residuais e redução do alerta em pacientes idosos ${ }^{27}$.

Em 2005, o FDA publicou uma recomendação advertindo o risco do uso da tiagabina em indicações além das descritas na bula, em virtude da ocorrência de crises epilépticas ${ }^{28}$.

\section{Antidepressivos}

\section{1) Doxepina}

Descoberta na Alemanha em 1963, a doxepina é um antidepressivo tricíclico que foi aprovado pelo FDA em 1974. Ao longo de muitos anos, tem se mostrado um antidepressivo seguro e efetivo em doses variando de $10-300 \mathrm{mg} /$ dia. A sedação parece ser um de seus efeitos colaterais mais comuns e dose-dependentes. A doxepina afeta múltiplos sistemas de neurotransmissores 
e, recentemente, o interesse nesse fármaco foi renovado no tratamento da insônia, pelo seu potente efeito antagonista H1 específico quando em baixas doses ${ }^{29}$.

Estudos recentes utilizaram a doxepina nas doses 1,3 e $6 \mathrm{mg}$ em pacientes adultos e idosos com insônia crônica primária e insônia transitória. Tais doses são menores que as utilizadas para tratamento de depressão, como ocorre com outros antidepressivos sedativos, tais como trazodona e mirtazapina. O medicamento foi bem tolerado, seguro e eficaz em todas as doses, produzindo melhora significativa subjetiva e objetiva do sono, com efeitos colaterais comparáveis ao placebo, sem efeitos residuais ou comprometimento de memória no dia seguinte ${ }^{29,30}$.

2) Agomelatina

A agomelatina, um novo antidepressivo com perfil farmacológico distinto, é um agonista dos receptores da melatonina MT1 e MT2, e apresenta efeito antagonista dos receptores serotoninérgicos $5-\mathrm{HT}_{2 C}{ }^{31}$. A agomelatina apresenta efeito de melhora na sincronização dos ritmos circadianos, o que poderia contribuir com a melhora do humor em pacientes com depressão ${ }^{32}$. A agomelatina diminui a latência para início do sono, o número de despertares, pode aumentar o sono de ondas lentas e a eficiência do sono ${ }^{33}$.

Ensaios clínicos demonstraram eficácia no tratamento da depressão em doses de 25 a $50 \mathrm{mg}$, com segurança, boa tolerabilidade e menor potencial de efeitos colaterais, tais como disfunção sexual ${ }^{34}$.

\section{Outras moléculas em desenvolvimento}

\section{1) Tasimelteon}

Tasimelteon é um agente crono-hipnótico em desenvolvimento e, assim como o ramelteon, é um agonista dos receptores MT1 e MT2 e está em fase 3 de investigação ${ }^{35}$.

\section{2) Gaboxadol}

Essa medicação ainda encontra-se em fase de testes e não tem aprovação para uso em insônia. Diferentemente dos hipnóticos não benzodiazepínicos, os quais são agonistas moduladores do GABA, pois agem em sítios alostéricos, induzindo mudanças na conformação do receptor GABA-A; o gaboxadol é propriamente um agonista, pois assim como o GABA, ele ativa diretamente os receptores GABA-A, constituindo-se assim numa nova classe de hipnóticos ${ }^{36}$. Resultados iniciais de estudos em idosos mostraram melhora da qualidade subjetiva do sono; entretanto, pode estar sujeito às mesmas restriçóes que a tiagabina em termos de segurança para pacientes com distúrbios do sono.

3) Eplivanserina

A eplivanserina é um novo agente, com novo perfil farmacológico, que vem sendo desenvolvido e testado no tratamento da insônia crônica. É um agonista inverso do subtipo $5-\mathrm{HT}_{2 \mathrm{~A}}$ da serotonina. De modo similar à trazodona, um antagonista $5 \mathrm{HT}_{2 \mathrm{~A}}$, a eplivanserina quase não tem afinidade para dopamina ou histamina. Além disso, parece não ter afinidade pelos receptores adrenérgicos. Atualmente, existem estudos clínicos de fase $3 \mathrm{em}$ andamento com o seu uso ${ }^{37,38}$.

\section{4) Almorexant}

É um novo agente que vem sendo avaliado com alguns resultados promissores no tratamento da insônia nos estudos pré-clínicos e de fase 2, promovendo o sono em animais e humanos. Trata-se de um antagonista dual dos receptores da orexina (OX1 e OX2). O sistema orexininérgico parece desempenhar um papel de destaque na promoção e manutenção da vigília, além de outras funçōes regulatórias. Reduçôes nos níveis de orexina podem estar associadas a um efeito promotor do sono ${ }^{39}$.

Os resultados destes primeiros estudos demonstraram que o almorexant reduziu o alerta e aumentou o tempo de sono em modelos animais e humanos, quando administrado durante a fase ativa do ciclo circadiano ${ }^{40,41}$.

\section{Discussão}

Uma vez que insônia é um transtorno crônico e necessita de tratamento de longo prazo, cresce a literatura e a busca para tais tratamentos, sejam eles medicamentosos ou comportamentais. Apesar de esta atualização focar na terapia medicamentosa da insônia, os autores reconhecem a importância da terapia cognitivocomportamental, que mostra melhora significativa nos sintomas da insônia, alem de exercer papel na manutenção da melhora após o tratamento medicamentoso ${ }^{2,42}$.

Observa-se a recente aprovação para uso clínico dos agonistas GABA-A, zolpidem MR e eszopiclone para uso em longo prazo para insônia, considerando aspectos de segurança. Entretanto, já se observava um aumento significativo do uso de antidepressivos sedativos entre pacientes com insônia, dentre eles a trazodona e mais raramente a mirtazapina ${ }^{43}$. Duas possíveis explicações seriam: primeiro, o fato de antidepressivos serem medicamentos utilizados para uso prolongado; segundo, mesmo que indicados em doses subterapêuticas para depressão, não se pode excluir alguma melhora no humor e ansiedade em tais pacientes, comorbidades comumente associadas à insônia crônica.

A busca de novos agonistas do receptor GABA-A, mais seletivos para o efeito sedativo-hipnótico, que se ligam à subunidade $\alpha 1 \mathrm{de}$ tal receptor e com meia-vida de eliminação ultracurta ou curta, de até cinco horas, considera aspectos de segurança e eficácia em médio e longo prazos, tendência atual da literatura para insônia crônica. Entretanto, estudos recentes apontam que algumas das drogas-z podem se ligar a outras subunidades que não somente $\alpha 1$. Além desses, os novos agonistas melatoninérgicos e novos antagonistas $5 \mathrm{HT}_{2}$ mostram que o efeito sedativo das diferentes medicaçôes investigadas dá-se por ativação ou inibição de distintos sistemas de neurotransmissão, o que pode determinar maior ou menor eficácia de cada um desses medicamentos. Novas evidências surgirão com a exploração de novos sistemas de neurotransmissão envolvidos na regulação vigília/sono, tais como a hipocretina, no tratamento da insônia.

\section{Agradecimentos}

Dalva Poyares e Sergio Tufik recebem financiamento do Conselho Nacional de Desenvolvimento Científico e Tecnológico (CNPq), Brasil. 
Financiamento e conflito de interesse

\begin{tabular}{|c|c|c|c|c|c|c|c|}
\hline $\begin{array}{l}\text { Membro do grupo } \\
\text { de autores }\end{array}$ & $\begin{array}{l}\text { Local de } \\
\text { trabalho }\end{array}$ & $\begin{array}{l}\text { Verba de } \\
\text { pesquisa }{ }^{1}\end{array}$ & $\begin{array}{c}\text { Outro apoio à } \\
\text { pesquisa ou educação } \\
\text { médica continuada }^{2}\end{array}$ & $\begin{array}{c}\text { Honorários } \\
\text { de } \\
\text { palestrantes }\end{array}$ & $\begin{array}{l}\text { Participação } \\
\text { acionária }\end{array}$ & $\begin{array}{l}\text { Consultorl } \\
\text { conselho } \\
\text { consultivo }\end{array}$ & Outro $^{3}$ \\
\hline Lucia Sukys Claudino & $\begin{array}{l}\text { HU-UFSC } \\
\text { HGCR } \\
\text { UNIPESP }\end{array}$ & - & - & - & - & - & - \\
\hline $\begin{array}{l}\text { Walter André dos } \\
\text { Santos Moares }\end{array}$ & UNIFESP & - & - & Ache $^{*}$ & - & - & - \\
\hline Sérgio Tufik & UNIFESP & $\begin{array}{l}\text { FAPESP }^{* *} \\
\text { CNPq }^{* *}\end{array}$ & - & - & - & - & - \\
\hline Dalva Poyares & UNIFESP & $\begin{array}{l}\text { FAPESP }^{* *} \\
\text { CNPq }^{* *}\end{array}$ & - & $\begin{array}{c}\text { Apsen* } \\
\text { Libbs }^{* *} \\
\text { Sanofi- } \\
\text { Aventis* } \\
\text { Ache* }^{*} \\
\text { Mantecorp* }\end{array}$ & - & - & - \\
\hline
\end{tabular}

* Modesto

** Significativa

*** Significativa. Montantes fornecidos à instituição do autor ou a colega onde o autor tem participação, não diretamente ao autor

Nota: HU-UFSC = Hospital Universitário, Universidade Federal de Santa Catarina; HGCR = Hospital Governador Celso Ramos-Santa Catarina, UNIFESP = Universidade Federal de São Paulo; FAPESP = Fundação de Amparo à Pesquisa do Estado de São Paulo; CNPq = Conselho Nacional de Desenvolvimento Científico e Tecnológico.

Mais informações, consultar as Instruções aos Autores.

Referências

1. Morin CM, LeBlanc M, Daley M, Gregoire JP, Merette C. Epidemiology of insomnia: prevalence, self-help treatments, consultations, and determinants of help-seeking behaviors. Sleep Med. 2006;7(2):123-30.

2. Schutte-Rodin S, Broch L, Buysse D, Dorsey C, Sateia M. Clinical guideline for the evaluation and management of chronic insomnia in adults. J Clin Sleep Med. 2008;4(5):487-504.

3. National Institutes of Health State of the Science Conference statement on Manifestations and Management of Chronic Insomnia in Adults, 2005 June 13-15. Sleep. 2005;28(9):1049-57.

4. McCall WV. A psychiatric perspective on insomnia. J Clin Psychiatry. 2001;62 Suppl 10:27-32.

5. Morphy H, Dunn KM, Lewis M, Boardman HF, Croft PR. Epidemiology of insomnia: a longitudinal study in a UK population. Sleep. 2007;30(3):274-80.

6. Rush CR. Behavioral pharmacology of zolpidem relative to benzodiazepines: a review. Pharmacol Biochem Behav. 1998;61(3):253-69.

7. Olubodun JO, Ochs HR, von Moltke LL, Roubenoff R, Hesse LM, Harmatz JS, Shader RI, Greenblatt DJ. Pharmacokinetic properties of zolpidem in elderly and young adults: possible modulation by testosterone in men. Br J Clin Pharmacol. 2003;56(3):297-304.

8. Dolder C, Nelson M, McKinsey J. Use of non-benzodiazepine hypnotics in the elderly: are all agents the same? CNS Drugs. 2007;21(5):389-405.

9. Staner L, Ertle S, Boeijinga P, Rinaudo G, Arnal MA, Muzet A, Luthringer R. Next-day residual effects of hypnotics in DSM-IV primary insomnia: a driving simulator study with simultaneous electroencephalogram monitoring. Psychopharmacology (Berl). 2005;181(4):790-8.

10. Hindmarch I, Patat A, Stanley N, Paty I, Rigney U. Residual effects of zaleplon and zolpidem following middle of the night administration five hours to one hour before awakening. Hum Psychopharmacol. 2001;16(2):159-67.

11. Krystal AD, Erman M, Zammit GK, Soubrane C, Roth T. Long-term efficacy and safety of zolpidem extended-release $12.5 \mathrm{mg}$, administered 3 to 7 nights per week for 24 weeks, in patients with chronic primary insomnia: a 6-month, randomized, double-blind, placebo-controlled, parallelgroup, multicenter study. Sleep. 2008;31(1):79-90.

12. Eszopiclone: esopiclone, estorra, S-zopiclone, zopiclone--Sepracor. Drugs $R$ D. 2005;6(2):111-5.

13. Hanson SM, Morlock EV, Satyshur KA, Czajkowski C. Structural requirements for eszopiclone and zolpidem binding to the gamma-aminobutyric acid type-A (GABAA) receptor are different. J Med Chemistry. 2008;51(22):7243-52.
14. Krystal AD, Walsh JK, Laska E, Caron J, Amato DA, Wessel TC, Roth T. Sustained efficacy of eszopiclone over 6 months of nightly treatment: results of a randomized, double-blind, placebo-controlled study in adults with chronic insomnia. Sleep. 2003;26(7):793-9.

15. Foster AC, Pelleymounter MA, Cullen MJ, Lewis D, Joppa M, Chen TK, Bozigian HP, Gross RS, Gogas KR. In vivo pharmacological characterization of indiplon, a novel pyrazolopyrimidine sedativehypnotic. J Pharmacol Exp Ther. 2004;311(2):547-59.

16. Rosenberg R, Roth T, Scharf MB, Lankford DA, Farber R. Efficacy and tolerability of indiplon in transient insomnia. J Clin Sleep Med. 2007;3(4):374-9.

17. Lankford A, Ancoli-Israel S. Indiplon: the development of a novel therapy for the treatment of sleep onset and sleep maintenance insomnia. Int J Clin Pract. 2007;61(6):1037-45.

18. Farber RH, Burke PJ. Post-bedtime dosing with indiplon in adults and the elderly: results from two placebo-controlled, active comparator crossover studies in healthy volunteers. Curr Med Res Opin. 2008;24(3):837-46.

19. Pandi-Perumal SR, Srinivasan V, Spence DW, Moscovitch A, Hardeland R, Brown GM, Cardinali DP. Ramelteon: a review of its therapeutic potential in sleep disorders. Adv Ther. 2009;26(6):613-26.

20. Sateia MJ, Kirby-Long P, Taylor JL. Efficacy and clinical safety of ramelteon: an evidence-based review. Sleep Med Rev. 2008;12(4):319-32.

21. Erman M, Seiden D, Zammit G, Sainati S, Zhang J. An efficacy, safety, and dose-response study of Ramelteon in patients with chronic primary insomnia. Sleep Med. 2006;7(1):17-24.

22. Mayer G, Wang-Weigand S, Roth-Schechter B, Lehmann R, Staner C, Partinen M. Efficacy and safety of 6-month nightly ramelteon administration in adults with chronic primary insomnia. Sleep. 2009;32(3):351-60.

23. Hardeland R. New approaches in the management of insomnia: weighing the advantages of prolonged-release melatonin and synthetic melatoninergic agonists. Neuropsychiatr Dis Treat. 2009;5:341-54.

24. Morgenthaler TI, Lee-Chiong T, Alessi C, Friedman L, Aurora RN, Boehlecke B, Brown T, Chesson AL Jr, Kapur V, Maganti R, Owens J, Pancer J, Swick TJ, Zak R; Standards of Practice Committee of the American Academy of Sleep Medicine. Practice parameters for the clinical evaluation and treatment of circadian rhythm sleep disorders. An American Academy of Sleep Medicine report. Sleep. 2007;30(11):1445-59.

25. Lemoine P, Nir T, Laudon M, Zisapel N. Prolonged-release melatonin improves sleep quality and morning alertness in insomnia patients aged 55 years and older and has no withdrawal effects. J Sleep Res. 2007;16(4):372-80.

26. Leach JP, Brodie MJ. Tiagabine. Lancet. 1998;351(9097):203-7. 
27. Walsh JK, Perlis M, Rosenthal M, Krystal A, Jiang J, Roth T. Tiagabine increases slow-wave sleep in a dose-dependent fashion without affecting traditional efficacy measures in adults with primary insomnia. $J$ Clin Sleep Med. 2006;2(1):35-41.

28. Flowers CM, Racoosin JA, Kortepeter C. Seizure activity and off-label use of tiagabine. N Engl J Med. 2006;354(7):773-4.

29. Goforth HW. Low-dose doxepin for the treatment of insomnia: emerging data. Expert Opin Pharmacother. 2009;10(10):1649-55.

30. Owen RT. Selective histamine H(1) antagonism: a novel approach to insomnia using low-dose doxepin. Drugs Today (Barc). 2009;45(4):261-7.

31. San L, Arranz B. Agomelatine: a novel mechanism of antidepressant action involving the melatonergic and the serotonergic system. Eur Psychiatry. 2008;23(6):396-402.

32. Lam RW. Sleep disturbances and depression: a challenge for antidepressants. Int Clin Psychopharmacology. 2006;21 Suppl 1:S25-9.

33. Quera Salva MA, Vanier B, Laredo J, Hartley S, Chapotot F, Moulin C, Lofaso F, Guilleminault C. Major depressive disorder, sleep EEG and agomelatine: an open-label study. Int J Neuropsychopharmacol. 2007; 10(5):691-6.

34. Zupancic M, Guilleminault C. Agomelatine: a preliminary review of a new antidepressant. CNS Drugs. 2006;20(12):981-92.

35. Hardeland R. Tasimelteon, a melatonin agonist for the treatment of insomnia and circadian rhythm sleep disorders. Curr Opin Investig Drugs. 2009;10(7):691-701.

36. Lancel M. Role of GABAA receptors in the regulation of sleep: initial sleep responses to peripherally administered modulators and agonists. Sleep. 1999;22(1):33-42.

37. Luthinger R, Delfolie A, Nicolas O, Brunet A, Pinquier J. Effects of Eplivanserin, a non-seletive agent, on driving and cognitive/ psychomotor performance: a randomized placebo-controlled trial in insomnia patients with difficulties maintaining sleep. 23th Annual Meeting of Associated Sleep Societies. Seattle: Sleep; 2009.

38. Erman M, Soubrane C, Morin CM, Hajak G. A randomized double-blind 12-week trial eplivanserin in insomniac patients with sleep mainttenance difficulties. 23th Annual Metting of the Associeted Sleep Societies. Seattle: Sleep; 2009.

39. Carter ME, Adamantidis A, Ohtsu H, Deisseroth K, de Lecea L. Sleep homeostasis modulates hypocretin-mediated sleep-to-wake transitions. J Neurosci. 2009;29(35):10939-49.

40. Malherbe P, Borroni E, Pinard E, Wettstein JG, Knoflach F. Biochemical and electrophysiological characterization of almorexant, a dual orexin 1 receptor (OX1)/orexin 2 receptor (OX2) antagonist: comparison with selective OX1 and OX2 antagonists. Mol Pharmacol. 2009;76(3):618-31.

41. Neubauer DN. Almorexant, a dual orexin receptor antagonist for the treatment of insomnia. Curr Opin Investig Drugs. 2010;11(1):101-10.

42. Associação Brasileira de Sono. Diretrizes para o diagnóstico e tratamento da insônia. Rio de Janeiro: Elsevier; 2009. p.114.

43. Wiegand $\mathrm{MH}$. Antidepressants for the treatment of insomnia: a suitable approach? Drugs. 2008;68(17):2411-7. 